\title{
Bulk observables and dileptons at SIS energies with a new transport approach SMASH
}

\section{Dmytro Oliinychenko*}

Frankfurt Institute for Advanced Studies, Ruth-Moufang-Str. 1, 60438 Frankfurt am Main, Germany

E-mail: oliiny@fias.uni-frankfurt.de

\section{Vinzent Steinberg, Jan Staudenmaier, Markus Mayer}

Frankfurt Institute for Advanced Studies, Ruth-Moufang-Strasse 1, 60438 Frankfurt am Main, Germany

Institute for Theoretical Physics, Goethe University, Max-von-Laue-Strasse 1, 60438 Frankfurt am Main, Germany

\section{Hannah Petersen}

Frankfurt Institute for Advanced Studies, Ruth-Moufang-Strasse 1, 60438 Frankfurt am Main, Germany

Institute for Theoretical Physics, Goethe University, Max-von-Laue-Strasse 1, 60438 Frankfurt am Main, Germany

GSI Helmholtzzentrum für Schwerionenforschung, Planckstr. 1, 64291 Darmstadt, Germany

Transport approaches are widely used to simulate heavy-ion collisions and allow to compute most experimental observables. In this contribution pion production, $v_{1}$ and $v_{2}$ of protons in $\mathrm{Au}+\mathrm{Au}$ collisions at energies $E_{\mathrm{lab}}=0.4-2 \mathrm{~A} \mathrm{GeV}$ and dilepton production in pp collisions at $\sqrt{s}=3.5$ $\mathrm{GeV}$ are computed using a new SMASH transport approach. These results are compared to the experimental data obtained by the FOPI and HADES collaborations. Overall, a good description of the data is achieved. Pion multiplicities and $N_{\pi^{-}} / N_{\pi^{+}}$ratio at $E_{\mathrm{lab}}=0.4-0.8 \mathrm{~A} \mathrm{GeV}$ are very sensitive not only to nuclear potentials, but also to Fermi motion and Pauli blocking effects. A good description of the proton $v_{1}$ and $v_{2}$ requires a nuclear potential corresponding to a hard equation of state.

The European Physical Society Conference on High Energy Physics

5-12 July

Venice, Italy

\footnotetext{
${ }^{*}$ Speaker.
} 


\section{Introduction}

In heavy-ion collisions at energies in the range $E_{\mathrm{lab}}=0.4-2 \mathrm{~A} \mathrm{GeV}$ excited, hot and baryonrich nuclear matter is produced, where interactions between hadrons mostly proceed via potentials and hadronic resonances. Transport simulations of such heavy-ion collisions allow to constrain resonance properties, as well as nuclear matter potentials, and to extract parameters of the hot nuclear matter Equation of State (EoS). Transport approaches also serve as a hadronic baseline for the search of the phase transition and the critical point, performed by the RHIC Beam Energy Scan [1] and the future experiments FAIR [2], NICA [3] and J-PARC [4]. Here we show the recent progress on simulations with the SMASH transport code [5] and compare SMASH results to selected bulk observables measured by the FOPI and HADES collaborations: pion production, proton flow and dilepton production. These simulations allow to validate and tune SMASH, preparing to make predictions for future experiments.

\section{Model description}

SMASH (Simulating Many Accelerated Strongly-interacting Hadrons) is a recently developed relativistic hadronic transport code. So far it has been tested against exact solutions of Boltzmann equation [6], served as a basis to implement "thermal bubbles" model, where forced thermalization is performed wherever the energy density is high enough [7], used to compute the viscosity of a hadron gas [8] and to study dilepton production [9]. Most of the well-established hadronic resonances published by the Particle Data Group [10] with masses up to $2.5 \mathrm{GeV}$ are implemented in SMASH. These hadrons interact via $2 \rightarrow 2$ elastic and inelastic collisions, resonance excitations and decays, potentials and string excitations. In the considered collision energy range of $E_{\text {lab }}=0.4-2$ $A \mathrm{GeV}$ string excitations are not happening. The rest of the reactions obey the detailed balance principle: matrix elements of forward and reverse reactions are equal. It allows to express unknown cross sections of $2 \rightarrow 2$ reverse reactions $\sigma_{c d \rightarrow a b}$ via the known $\sigma_{a b \rightarrow c d}$, as well as cross sections of resonance formation $\sigma_{a b \rightarrow R}(\sqrt{s})$ via the partial resonance width $\Gamma_{R \rightarrow a b}\left(m_{R}\right)$. Explicit expressions are given in [5]. All the resonances obey a relativistic Breit-Wigner spectral function with mass-dependent widths computed using the Manley-Saleski formalism [11]. The mentioned "known" $2 \rightarrow 2$ cross sections $N N \rightarrow N \Delta, N N \rightarrow N N^{*}, N N \rightarrow \Delta \Delta$ and strangeness exchange reactions are either parametrized directly to match experimental data or their matrix elements are parametrized.

From reaction to reaction hadrons are propagating according to the equations of motion following the one-particle Hamiltonian

$$
H_{i}\left(p_{i}, r_{i}\right)=\sqrt{\vec{p}_{i}^{2}+m_{\mathrm{eff}}^{2}}+U\left(\vec{r}_{i}\right),
$$

where $m_{\mathrm{eff}}$ is the mass for stable hadrons and the effective mass for resonances in accordance with their mass distribution (e.g. Breit-Wigner). At this point, the potential depends only on the coordinates, but not on the momentum of the particles and has the following form

$$
U\left(\rho, \rho_{I 3}\right)=a\left(\rho / \rho_{0}\right)+b\left(\rho / \rho_{0}\right)^{\tau} \pm 2 S_{\mathrm{pot}} \frac{\rho_{I 3}}{\rho_{0}} .
$$


Bulk observables and dileptons at SIS energies with a new transport approach SMASH Dmytro Oliinychenko

\begin{tabular}{cccccc}
\hline & $a[\mathrm{MeV}]$ & $b[\mathrm{MeV}]$ & $\tau$ & $S_{\text {pot }}[\mathrm{MeV}]$ & $K[\mathrm{MeV}]$ \\
\hline default & -209.2 & 156.4 & 1.35 & 18 & 240 \\
soft & -356.0 & 303.0 & 1.17 & 18 & 200 \\
hard & -124.0 & 71.0 & 2.0 & 18 & 380 \\
\hline
\end{tabular}

Table 1: Parameters of potential in Eq. (2.2). $K$ is corresponding isothermal compressibility.

Here $\rho$ is the Eckart rest frame baryon density and $\rho_{I 3}$ is the Eckart rest frame baryon isospin density of the relative isospin projection $I_{3} / I$. It follows from the above Hamiltonian and potential that SMASH is a BUU-type code (see [12] for details), where energy and momentum are only conserved over the event average. Parameters of the potential are given in Table 1 for different compressibilities. The "default" parameters were agreed on for a recent transport code comparison [12]. This simple potential does not include momentum-dependent terms and Coulomb interaction, but it allows to understand the influence of Skyrme parameters on the bulk observables and validate the code in a simple setup.

In SMASH nuclei are initialized according to the Woods-Saxon distribution assuming independent nucleons, $\rho(\vec{r}) / \rho_{0}=\left(1+e^{\frac{r-R}{d}}\right)^{-1}$. In momentum space the Local Density Approximation is adopted, where the momenta of nucleons are sampled from a Fermi sphere of radius $p_{F}(\vec{r})=\hbar c\left(3 \pi^{2} \rho(\vec{r})\right)^{1 / 3}$ in the rest frame of the nucleus. The fermionic nature of nucleons is accounted for in SMASH via Pauli blocking: a reaction with fermions in the final state is rejected with probability $1-\prod_{i}\left(1-f_{i}\right)$ (see [13] and references therein for an explanation), where the product is over the final-state fermions and $f_{i}$ are the phase-space densities of the same species at the positions of outgoing particles in the reaction. In SMASH $f_{i}(\vec{r}, \vec{p})$ is estimated by counting particles in a momentum-space sphere of radius $80 \mathrm{MeV}$ around $\vec{p}$ and a weighted counting in a coordinate space sphere of radius $2.2 \mathrm{fm}$ around $\vec{r}$. The weights are described in detail in the appendix of [5]. SMASH takes advantage of the test-particles ansatz: $N \rightarrow N \cdot N_{\text {test }}$ particles are sampled and all the cross sections are scaled as $\sigma \rightarrow \sigma / N_{\text {test }}$.

\section{Bulk observables and equation of state}

In heavy-ion collisions the effects of potentials, Fermi motion and Pauli blocking are acting simultaneously and cannot be disentangled. However, in transport simulations it is possible to add these effects sequentially and observe their influence as shown in Fig. 1. We start by a so-called cascade simulation of $\mathrm{Au}+\mathrm{Au}$ collisions at $E_{\text {lab }}=0.4-2 \mathrm{~A} \mathrm{GeV}$, where potentials, Fermi-motion and Pauli blocking are off. The centrality selection matches the FOPI experimental procedure (see appendix $\mathrm{C}$ of [5] for details). As one can see in Fig. 1, this is enough to reproduce pion multiplicities and $N_{\pi^{-}} / N_{\pi^{+}}$ratio at the beam energies above $0.8 \mathrm{GeV}$ reasonably well. The inclusion of test-particles with $N_{\text {test }}=20$ does not alter the results of the cascade significantly. Switching on potentials with the "default" parameters from Table 1 reduces total pion multiplicities and reduces the $N_{\pi^{-}} / N_{\pi^{+}}$ratio at $E_{\text {lab }}=0.4-0.6 \mathrm{~A} \mathrm{GeV}$. The potential does not let slower nucleons come close enough to collide and form resonances, which can be a reason of the reduced pion multiplicities. The change of the ratio reflects the effect of the symmetry term in the potential. Introducing Fermi motion adds energy to the colliding nucleons, increasing the pion multiplicity and reducing the rel- 

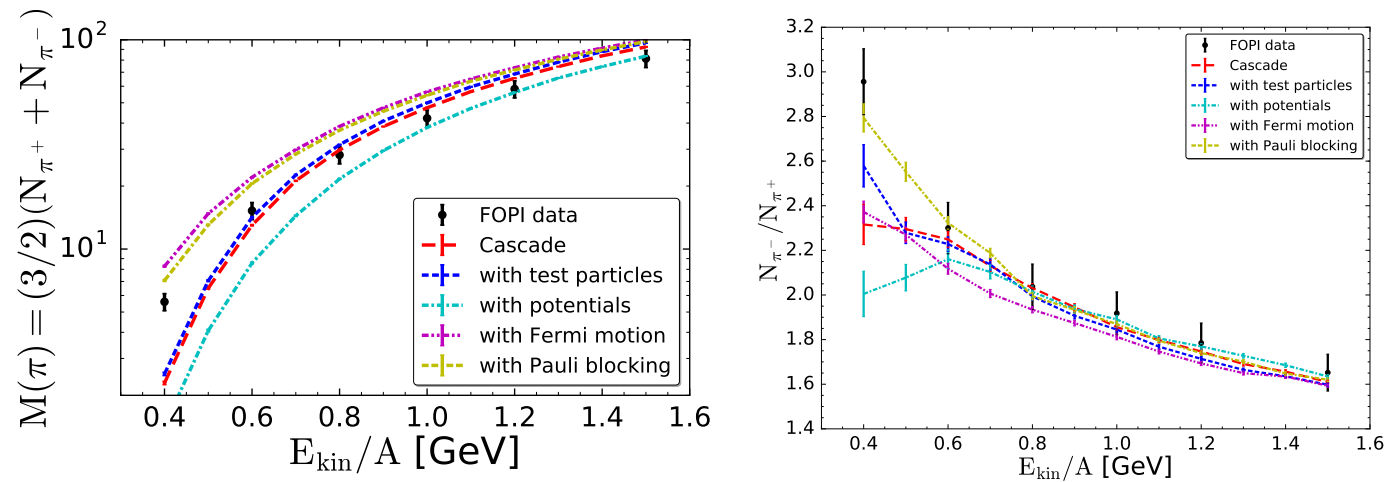

Figure 1: Total pion multiplicity (left panel) and $N_{\pi^{-}} / N_{\pi^{+}}$ratio (right panel) in Au+Au collisions at energies $E_{\text {lab }}=0.4-2 \mathrm{~A} \mathrm{GeV}$ from SMASH with sequentially added features: testparticles, potentials, Fermi motion and Pauli blocking. The calculation is compared to the data from FOPI [17].

ative effect of the symmetry term in the potential. Finally, Pauli blocking prevents some reactions to occur and thus reduces the total pion multiplicity. The $N_{\pi^{-}} / N_{\pi^{+}}$ratio at $E_{\mathrm{lab}}=0.4-0.6 \mathrm{~A} \mathrm{GeV}$ is enhanced by the Pauli-blocking. In this energy range the effects of potentials, Fermi motion and Pauli blocking are equally important for $N_{\pi^{-}} / N_{\pi^{+}}$ratio. Additional constraints can be provided by other measurements.
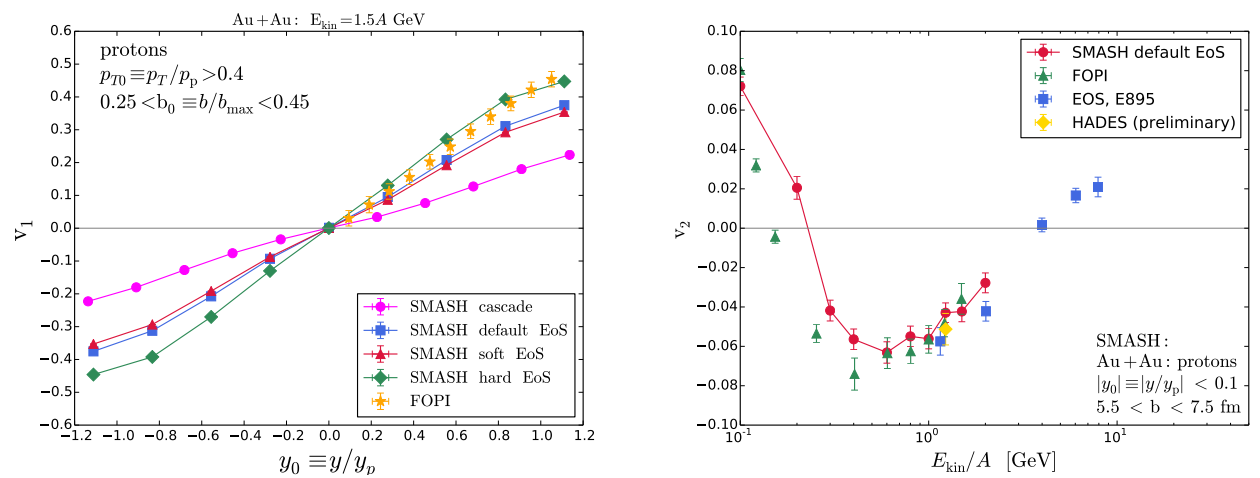

Figure 2: Directed flow of protons $v_{1}$ versus rapidity at $E_{\mathrm{lab}}=1.5 \mathrm{~A} \mathrm{GeV}$ (left panel) and integrated elliptic flow of protons $v_{2}$ versus collision energy (right panel).

One such measurement is the proton flow measured by FOPI [18, 19], EoS and E895 [21] and HADES [22] collaborations. The directed flow $v_{1}=\left\langle\frac{p_{x}}{p_{T}}\right\rangle$ (the brackets $\langle\ldots\rangle$ indicate an average over all particles and events) measures whether the participants go with or against the spectators. In Fig. 2 the directed flow of protons is shown for potentials corresponding to different isothermal compressibilities $K$ of nuclear matter. The harder the equation of state, the more repulsive the potential acts and the larger slope $d v_{1} / d y$ is produced, as shown in the left panel of Fig. 2. However, note that all protons are used for calculation, i.e. the clusterization of protons to deuterons and other light nuclei is not taken into account. Since the hard equation of state reproduces the FOPI data best, it is used to compare the integrated elliptic flow of SMASH with experimental data. The elliptic flow is calculated via $v_{2}=\left\langle\frac{p_{x}^{2}-p_{y}^{2}}{p_{T}^{2}}\right\rangle$. At beam energies less than $4 \mathrm{~A} \mathrm{GeV}$ the spectators 
are so slow that they block the emission of particles in $\mathrm{x}$-direction. Thus, most of the participants are squeezed out in y-direction leading to a negative $v_{2}$. At higher energies the spectators are gone fast enough and particles can also be emitted in X-direction. In this case positive $v_{2}$ is produced, explained by larger pressure gradients in $\mathrm{x}$-direction. SMASH is able to reproduce the integrated $v_{2}$ of protons at $E_{\text {lab }}>0.8 \mathrm{GeV}$, while at lower energies the trend is correct, but the value of $v_{2}$ is overestimated. The description of $v_{1}(y)$, integrated $v_{2}$ and $v_{2}(y)$ (not shown in this contribution) consistently requires a hard potential, which is in agreement with previous calculations with UrQMD [15], but in contrast to the IQMD result [16]. This (dis-)agreement can however be coincidental due to absence of clustering and the simplified potential in this work. Also the different amount of high mass resonances might influence these results.

\section{Dilepton production}

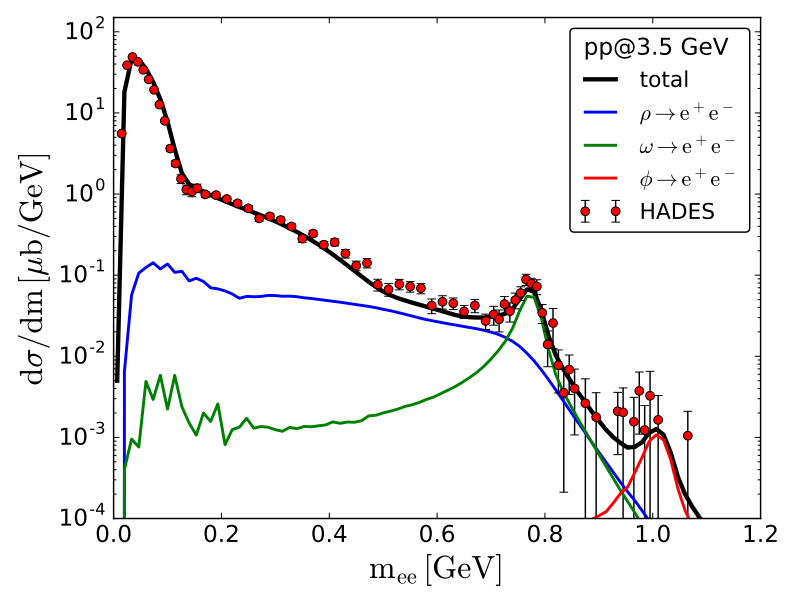

Figure 3: Total dilepton spectra in pp collisions from SMASH compared to HADES data [20]. Contributions from $\rho, \omega$ and $\phi$ decays are shown separately. Contributions from $\Delta, \eta$, as well as $\pi, \omega$ and $\eta$ Dalitz decays are not shown separately, but enter the total spectrum.

A potential source of uncertainty in a transport calculation originates from the treatment of the resonances, especially far from the pole mass. Resonance properties are validated by comparing the dilepton production in SMASH to experimental results. Dileptons in SMASH are treated pertubatively [14] and produced through decays of the $\pi, \eta, \eta^{\prime}, \rho, \omega, \phi$ and $\Delta$. In Fig. 3 dilepton invariant mass spectrum from SMASH is compared to the one from the HADES collaboration [20]. The (vacuum) treatment of resonances in SMASH is sufficient to reproduce dilepton spectra in pp collisions and small systems [23]. Notable are contributions below the hadronic thresholds for the direct vector meson decay channels, which stem from the proper inclusion of the dilepton decays into the calculation of the spectral function. Based on the agreement with experimental data for smaller system, the effect of in-medium modifications for resonances can be studied for larger systems [23].

\section{Summary and outlook}

Selected results on pion production and proton flow in $E_{\mathrm{lab}}=0.4-2 \mathrm{~A} \mathrm{GeV} \mathrm{Au}+\mathrm{Au}$ collisions 
and dilepton production in pp collisions at $\sqrt{s}=3.5 \mathrm{GeV}$ were used to validate SMASH transport approach. It was demonstrated that for pion production at these energies potentials are as important as Fermi motion and Pauli blocking. Proton $v_{1}$ and $v_{2}$ are described well by a calculation with a hard potential. As an outlook, these studies should be extended to a larger set of observables, accounting for proton clustering and more advanced potentials, including Coulomb and momentum-dependent terms.

\section{Acknowledgments}

The authors acknowledge funding of a Helmholtz Young Investigator Group VH-NG-822 from the Helmholtz Association and GSI. This work was supported by the Helmholtz International Center for the Facility for Antiproton and Ion Research (HIC for FAIR) within the framework of the Landes-Offensive zur Entwicklung Wissenschaftlich-Ökonomischer Exzellenz (LOEWE) program launched by the State of Hesse. V. S., J. S. and M. M. acknowledge support by the Helmholtz Graduate School for Hadron and Ion Research (HGS-HIRe).

\section{References}

[1] M. M. Aggarwal et al. [STAR Collaboration], arXiv:1007.2613 [nucl-ex].

[2] T. Ablyazimov et al. [CBM Collaboration], Eur. Phys. J. A 53, no. 3, 60 (2017) [arXiv:1607.01487 [nucl-ex]].

[3] V. Kekelidze, R. Lednicky, V. Matveev, I. Meshkov, A. Sorin and G. Trubnikov, Phys. Part. Nucl. Lett. 9, 313 (2012).

[4] H. Sako et al., Nucl. Phys. A 931, 1158 (2014).

[5] J. Weil et al., Phys. Rev. C 94, no. 5, 054905 (2016) [arXiv:1606.06642 [nucl-th]].

[6] J. Tindall, J. M. Torres-Rincon, J. B. Rose and H. Petersen, Phys. Lett. B 770, 532 (2017) [arXiv:1612.06436 [hep-ph]].

[7] D. Oliinychenko and H. Petersen, J. Phys. G 44, no. 3, 034001 (2017) [arXiv:1609.01087 [nucl-th]].

[8] J.-B. Rose, J. M. Torres-Rincon, A. Schäfer, D. R. Oliinychenko and H. Petersen, arXiv:1709.03826 [nucl-th].

[9] J. Weil, J. Staudenmaier and H. Petersen, J. Phys. Conf. Ser. 742, no. 1, 012034 (2016) [arXiv:1604.07028 [nucl-th]].

[10] C. Patrignani et al. [Particle Data Group], Chin. Phys. C 40, no. 10, 100001 (2016).

[11] D. M. Manley and E. M. Saleski, Phys. Rev. D 45, 4002 (1992). doi:10.1103/PhysRevD.45.4002

[12] J. Xu et al., Phys. Rev. C 93, no. 4, 044609 (2016) doi:10.1103/PhysRevC.93.044609 [arXiv:1603.08149 [nucl-th]].

[13] S. Pratt and W. Bauer, Phys. Lett. B 329, 413 (1994) doi:10.1016/0370-2693(94)91083-9 [nucl-th/9404027].

[14] K. Schmidt, E. Santini, S. Vogel, C. Sturm, M. Bleicher and H. Stocker, Phys. Rev. C 79, 064908 (2009) [arXiv:0811.4073 [nucl-th]]. 
[15] H. Petersen, Q. Li, X. Zhu and M. Bleicher, Phys. Rev. C 74, 064908 (2006) doi:10.1103/PhysRevC.74.064908 [hep-ph/0608189].

[16] A. Le Fèvre, Y. Leifels, W. Reisdorf, J. Aichelin and C. Hartnack, Nucl. Phys. A 945, 112 (2016) doi:10.1016/j.nuclphysa.2015.09.015 [arXiv:1501.05246 [nucl-ex]].

[17] W. Reisdorf et al. [FOPI Collaboration], Nucl. Phys. A 781, 459 (2007) [nucl-ex/0610025].

[18] W. Reisdorf et al. [FOPI Collaboration], Nucl. Phys. A 876, 1 (2012) [arXiv:1112.3180 [nucl-ex]].

[19] A. Andronic et al. [FOPI Collaboration], Phys. Lett. B 612, 173 (2005) [nucl-ex/0411024].

[20] G. Agakishiev et al. [HADES Collaboration], Eur. Phys. J. A 48, 64 (2012) [arXiv:1112.3607 [nucl-ex]].

[21] C. Pinkenburg et al. [E895 Collaboration], Phys. Rev. Lett. 83, 1295 (1999) [nucl-ex/9903010].

[22] B. Kardan, J. Phys. Conf. Ser. 742, no. 1, 012008 (2016). doi:10.1088/1742-6596/742/1/012008

[23] J. Staudenmaier, J. Weil, V. Steinberg, H. Petersen, in preparation 